\title{
Getting a free ride on poultry farms: how highly pathogenic avian influenza may persist in spite of its virulence
}

\author{
Giulio Alessandro De Leo $•$ Luca Bolzoni
}

Received: 15 October 2010 / Accepted: 17 January 2011 /Published online: 8 September 2011

(C) The Author(s) 2011. This article is published with open access at Springerlink.com

\begin{abstract}
It is well-known that highly pathogenic avian influenza (HPAI) strains can arise from low pathogenic strains (LPAI) during epidemics in poultry farms. Despite this, the possibility that partial cross-immunity triggered by previous exposure to LPAI viruses may reduce the pathogenicity of HPAI and thus enhance its persistence has been generally overlooked in both empirical and theoretical work on avian influenza. We propose a simple mathematical model to investigate the interacting dynamics of HPAI and LPAI strains of avian influenza in small-scale poultry farms. Through the analysis of a deterministic ordinary differential equations model, we show that: (1) for a wide range of realistic model parameters, the reduction in pathogenicity yielded by previous LPAI infection might allow an HPAI strain that would not be able to persist in a host population when alone $\left(\mathfrak{R}_{0}<1\right)$ to invade and co-exist in the host population along with the LPAI strain and (2) the coexistence between the HPAI and LPAI strains may be characterized by multiyear periodicity. Because simulations showed that troughs between epidemics can be deep, with only a fraction of existing flocks infected by the HPAI strain, we also ran an individual-based stochastic version of the dynamical model to analyze the potential for natural
\end{abstract}

Giulio Alessandro De Leo and Luca Bolzoni contributed equally to this study

G. A. De Leo $(\bowtie) \cdot$ L. Bolzoni

Dipartimento di Scienze Ambientali,

Università degli Studi di Parma,

Viale Usberti 11/A,

43100 Parma, Italy

e-mail: giulio.deleo@unipr.it

\section{Bolzoni}

Environment and Natural Resources Area, Research and

Innovation Centre, Foundation Edmund Mach,

S. Michele all'Adige, TN, Italy fade-out of the HPAI strain. The analysis of the stochastic model confirms the prediction that previous exposure to a LPAI strain can significantly increase the duration of the epidemics by an HPAI strain before it fades from the population.

Keywords Infectious diseases - Avian influenza . Multiple strain dynamics · Coexistence · Cross-immunity . Poultry farms

\section{Introduction}

The avian influenza A virus (AI) is a zoonotic pathogen capable of infecting a wide variety of gallinococcus poultry, captive birds, and free-ranging wild bird species under natural and experimental conditions. While waterfowlsuch as wild ducks, gulls, and shorebirds - are the main AI reservoirs, domestic birds have historically been the source of cross-species transmission to humans and the cause of consequent pandemic events (Webster et al. 1992). The best-known example of transmission to humans is the H5N1-type avian virus that circulated in Southeast Asia in the early 2000s (Webster et al. 2006). A better understanding of the ecology of avian influenza viruses is therefore crucial for developing effective control of future influenza pandemics.

AI viruses can be classified on the basis of two surface antigens, the hemagglutining (HA), of which there are 16 subtypes (H1-H16) and the neuraminidase (NA), of which there are 9 subtypes (N1-N9). The majority of the resulting 144 combinations is maintained in aquatic bird populations and causes no signs of diseases. The influenza strains causing mild pathogenicity in domestic birds are called low pathogenic avian influenza (LPAI) viruses. Viruses of the 
H5 and H7 subtypes may cause highly pathogenic avian influenza in chickens with up to $100 \%$ mortality only few days after infection. These strains are therefore called high pathogenic avian influenza (HPAI) viruses.

HPAI viruses are likely to emerge through mutation or, more likely, reassortment of LPAI viruses on poultry farms (Alexander 2007). Results of phylogenetic analysis of the H7 subtype show that HPAI viruses do not constitute a separate lineage but seem to arise from LPAI strains (Banks et al. 2000). The emergence of HPAI during outbreaks of LPAI strains has been confirmed in several cases on poultry farms worldwide. An example is the 1983 H5N2 LPAI outbreak chicken farms in Pennsylvania (USA). The epidemics started with an LPAI virus that caused low mortality in poultry farms. Six months later, the same virus had mutated to become highly pathogenic causing greater than $80 \%$ mortality among birds (Bean et al. 1985). Such a transformation event also occurred recently in Italy, where an H7N1 avian influenza epidemic began as an LPAI in April 1999 and mutated to become HPAI in December of the same year (Capua et al. 2000). Comparable progressions of HPAI virus have been recorded in Mexico (Garcia et al. 1996), Pakistan (Naeem 1998), Chile (Rojas et al. 2002), the Netherlands (Elbers et al. 2004), and Canada (Bowes et al. 2004). The virus can occasionally spill over from farms to wild bird populations, where HPAI is believed to be unable to sustain itself (Takekawa et al. 2010). As illustrated by Truscott et al. (2007), HPAI virus may eventually fade out from a sparse network of poultry farms after the first epidemic outbreak.

In the past few years, however, a heightened occurrence of HPAI has been noted, especially in Southeast Asia, where it is now considered endemic (Chen et al. 2004; Li et al. 2004). The possibility of its circulation into free-ranging bird species or its spread through highly migratory birds has been also considered (Takekawa et al. 2010). Despite years of empirical data, laboratory experiments, and theoretical works, the detailed mechanisms of continuous emergence and persistence of HPAI viruses - both in free-range, backyards farms and intensive poultry production facilities - are still controversial and poorly understood.

Two main hypotheses have been formulated to explain why HPAI viruses have recently tended to become endemic despite their high pathogenicity. The first hypothesis is that HPAI virus might be able to persist in the external environment (Takekawa et al. 2010). Theoretical analyses by Roche et al. (2005), Rohani et al. (2009), and some experimental studies (Stallknecht and Brown 2008; Brown et al. 2007) indicated that some HPAI H5N1 genotypes may persist long enough in the environment to become part of the endemic cycles of viruses. The second hypothesis is that partial cross-immunity triggered by previous exposure reduces the pathogenicity of HPAI. In this case, infection by HPAI causes a milder disease in domestic birds, thus increasing the duration of the epidemics and, in turn, the chance that the virus is transmitted to other susceptible farms. Potential for cross-protection has been observed both in controlled and natural conditions. For instance, clinical studies analyzed in vitro the interaction between different avian influenza subtypes and strains. Van der Goot et al. (2008) and Poetri et al. (2009) showed that vaccines produced from an $\mathrm{H} 5 \mathrm{~N} 2$ influenza subtype can protect ducks and chickens against severe illness and are able to reduce susceptibility toward a genetically and antigenically distant $\mathrm{H} 5 \mathrm{~N} 1$ subtype. These results indicate that cross-protection is expected not only between influenza strains within the same subtype but also between different subtypes. Moreover, laboratory experiments show that hosts previously infected with LPAI H5N2 virus can survive an infection with HPAI H5N2 virus or can directly escape the infection (van der Goot et al. 2003), while fully susceptible animals exposed to HPAI virus invariably die after infection (Swayne and Halvorson 2003; van der Goot et al. 2003, 2005). Similarly, Kalthoff et al. (2008) showed that naïve mute swans died shortly after infection with HPAI H5N1 but those with previous exposure to LPAI viruses were able to survive without apparent symptoms. Fereidouni et al. (2009) also showed that prior infection with low pathogenic $\mathrm{H} 5 \mathrm{~N} 2$ provided homosubtype immunity against HPAI H5N1 in waterfowl. These studies imply that previous infection with LPAI virus effectively reduces susceptibility of the host to infection and decreases transmission of HPAI virus, affecting the course of HPAI not only clinically, but also epidemiologically. Therefore, the presence of LPAI in the host population can have a twofold effect (Mannelli et al. 2007). On the one hand, it can increase the HPAI persistence by lengthening the infectious period. On the other hand, it can decrease HPAI persistence by reducing host susceptibility and, consequently, the probability of new infections. When effects on survival of diseased individuals overwhelm those on transmission between infected and susceptible, there is the possibility that unfit HPAI strains unable to spread in naïve populations may persist in the presence of LPAI.

While these two hypotheses (i.e., environmental transmission versus cross-protection) are not mutually exclusive and several factors or processes probably contribute to the emergence and persistence of HPAI, the possibility that partial cross-immunity triggered by previous exposure to LPAI viruses may reduce the pathogenicity of HPAI has been generally overlooked in both empirical and theoretical work of avian influenza (Webster et al. 2006). The present work was 
specifically aimed at addressing the second of these hypotheses and was designed to test whether and under what conditions cross-protection provided by previous exposure to LPAI infection may permit the persistence of an HPAI strain that otherwise would rapidly fade out from the population. The analysis was performed by extending the classical susceptible-infected-recovered (SIR) framework into a deterministic system of ordinary differential equations designed to describe the joint dynamics of HPAI and LPAI viruses and to account for the reduced pathogenicity of HPAI infections in flocks previously exposed to LPAI strains. Stability and transient dynamics of the two-strain model were investigated through a bifurcation analysis with respect to a realistic range of possible values of model parameters.

Because the prevalence of HPAI may be very low between outbreaks, we also developed a stochastic version of the model (as in Stollenwerk et al. 2004) to explicitly account for the possibility of a natural fade-out of the pathogen after an outbreak. In fact, stochastic models of infectious disease dynamics have shown that even pathogens that would be able to steadily persist in a population in a deterministic framework can go extinct by chance as a consequence of demographic stochasticity. In the past decade, several studies have explored the interaction between strain diversity and epidemiological dynamics in stochastic frameworks (van der Goot et al. 2003; Abu-Raddad and Ferguson 2004; Kirupaharan and Allen 2004; Stollenwerk et al. 2004; Truscott et al. 2007; Restif and Grenfell 2006, 2007), but to our knowledge this is the first attempt to address the epidemiological consequences of partial cross-immunity in the emergence of highly pathogenic avian influenza strains. The results of the stochastic analysis were thus compared to those of the deterministic version of the model to check for consistency and differences between the two approaches.

After Bicknell et al. (1999) and Elbakidze (2008), we applied our analysis to a homogeneous population of backyard farms where the unit of observation is represented by the flock/premise. We chose a flock-based model (instead of an individual-based model) because the spread of infections in domestic animals is more suitably described by farm-to-farm than animal-to-animal transmission (e.g., Keeling et al. 2003; Tildesley et al. 2006; Mulatti et al. 2010). Moreover, within-farm dynamics during influenza epidemics are significantly faster than between-farm dynamics (Truscott et al. 2007). As a consequence, it is an acceptable approximation to neglect within-farm dynamics in the model. In the specific case of avian influenza, epidemiological investigations of outbreaks in the USA reveal that the most likely source of infection for domestic farms is bird-to-bird contact at live birds markets (Garnett 1987; Pelzel et al. 2006), though the possibility of infection through feed or water contamination, cages, and other equipment cannot be ruled out. Moreover, several other studies have emphasized the central role of backyard poultry flocks in the spread of HPAI virus, both in developed (e.g., the Netherlands; Bavinck et al. 2009) and developing countries (e.g., Vietnam; Henning et al. 2009). Small poultry farms, such as backyard flocks, comprise a substantial proportion of the total number of poultry farms in developed countries (more than $94 \%$ of US farms have fewer than 100 birds; Elbakidze 2008). It is therefore important to concentrate the analysis of avian influenza dynamics on small-scale farms, which frequently do not follow to the strict sanitary and biosecurity regulations (such as isolation and disinfection) applied on large industrial farms.

\section{Deterministic model}

We expand the traditional SIR framework with autonomous ordinary differential equations to model the effect of LPAI on HPAI outbreaks on small poultry farms.

Following Iwami et al. (2007), the dynamics of the HPAI virus when it is isolated (that is, in the absence of circulation of an LPAI variant) are described by the following equations:

$$
\begin{aligned}
& \dot{S}=c-\mu S-\beta_{H} S I_{H} \\
& \dot{I}_{H}=\beta_{H} S I_{H}-\left(\mu+\alpha_{H}\right) I_{H}
\end{aligned}
$$

where, $S$ and $I$ are the number of susceptible and infected backyard poultry farms (flocks), respectively; $c$ is the flock restocking rate [number year ${ }^{-1}$ ]; $\mu$ is the rate of flock removal, either because the birds of a flock are sold in the market or because they die due to causes other then avian influenza; $\beta_{H}$ the transmission rate between infected and susceptible farms; and $\alpha_{H}$ is the additional mortality rate caused by the HPAI virus.

We have thus assumed that between-flock HPAI transmission occurs at a rate that is linearly related to the number of infected flocks. As a consequence, the contact term is given by the force of infection $\lambda=\beta_{H} I_{H}$ times the number of susceptible farms. The HPAI flock infectious period is assumed to be exponentially distributed with mean durations of $\left(\mu+\alpha_{H}\right)^{-1}$. It is assumed that flocks infected with the HPAI virus do not recover and all the birds eventually die.

The dynamics of the LPAI virus when isolated (that is, in the absence of circulation of an HPAI variant) differ from that of the HPAI in isolation because LPAI viruses are assumed to cause negligible disease-induced mortality for birds. As a consequence, almost all birds infected with LPAI survive the influenza infection and may develop 
immunity. The LPAI dynamics in isolation can be thus described by the following SIR model:

$\dot{S}=c-\mu S-\beta_{L} S I_{L}$

$\dot{I}_{L}=\beta_{L} S I_{L}-\left(\mu+\delta_{L}\right) I_{L}$

$\dot{R}_{L}=\delta_{L} I_{L}-\mu R_{L}$

where, $I_{L}$ and $R_{L}$ are the LPAI-infected and recovered flocks, respectively, $\beta_{L}$ is the between-flock transmission rate, $\delta_{L}$ is the LPAI recovery rate, and $\left(\mu+\delta_{L}\right)^{-1}$ is the mean flock infectious period. We do not include loss of immunity processes in the model since the life expectancy of domestic poultry (about 100 days) is lower than the estimated immunity period against influenza (about 6 months).

We then assumed that LPAI virus may partially protect hosts against HPAI strain by acquired immunity as shown by van der Goot et al. (2003), Pantin-Jackwood and Swayne (2009), Kalthoff et al. (2008), and Fereidouni et al. (2009). We also assumed that infected birds with HPAI strains cannot be infected with LPAI strains since the HPAI infectious period is too short and the low pathogenicity strain cannot take over an HPAI-infected host before the host dies (Stegeman et al. 2004; Tiensin et al. 2007). Consequently, individuals recovered from LPAI that are then infected with HPAI virus may develop a mild form of highly pathogenic influenza characterized by either a lower level of transmission and/or virulence than the full-blown infection (Fig. 1). This new class of mildly infected individuals was described in the model as new mild infections $\left(I_{M}\right)$. Then, a fraction of these individuals may recover, developing immunity to both strains and moving into class $R_{M}$. When both the LPAI virus and the HPAI virus are circulating among the poultry farms at the same time, the epidemiological dynamics can be described by the following system of equations:

$$
\begin{aligned}
& \dot{S}=c-\mu S-\beta_{L} S I_{L}-\beta_{H} S\left(I_{H}+I_{M}\right) \\
& \dot{I}_{H}=\beta_{H} S\left(I_{H}+I_{M}\right)-\left(\mu+\alpha_{H}\right) I_{H} \\
& \dot{I}_{L}=\beta_{L} S I_{L}-\left(\mu+\delta_{L}\right) I_{L} \\
& \dot{R}_{L}=\delta_{L} I_{L}-\mu R_{L}-\sigma \beta_{H} R_{L}\left(I_{H}+I_{M}\right) \\
& \dot{I}_{M}=\sigma \beta_{H} R_{L}\left(I_{H}+I_{M}\right)-\left(\mu+\alpha_{M}+\delta_{M}\right) I_{M} \\
& \dot{R}_{M}=\delta_{M} I_{M}-\mu R_{M}
\end{aligned}
$$

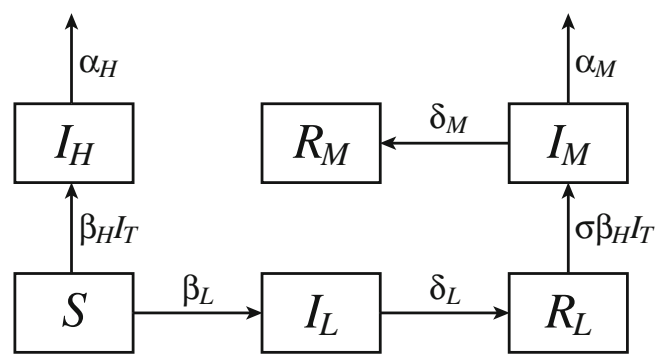

Fig. 1 General flow chart of the HPAI-LPAI model (3). Where $I_{T}=I_{H}+I_{M}$ where, $\alpha_{M}$ is the additional death rate caused by mild forms of avian influenza $\left(<<\alpha_{H}\right), \delta_{M}$ is the mild form recovery rate, $\left(\mu+\alpha_{M}+\delta_{M}\right)^{-1}$ is the mean infectious period in flock carrying mild infections, and $\sigma$ is the susceptibility reduction for individuals previously infected with LPAI $\left(R_{L}\right)$. As discussed by Grenfell (2001), there may exist an evolutionary trade-off between the duration of infection (specifically, the additional death rate $\alpha_{H}$ ) and susceptibility, the lower the transmission rate $(\sigma \beta)$ the higher the duration of the infection. Accordingly, we have assumed $\sigma<1$ and $\left(\mu+\alpha_{M}+\delta_{M}\right)^{-1}>\left(\mu+\alpha_{H}\right)^{-1}$.

\section{Stochastic model}

Although ordinary differential equation (ODE) models are useful tools for understanding the dynamics of pathogen persistence, the treatment of flocks as continuous entities can produce unrealistic results, such as an extremely low number of infected flocks during inter-epidemic periods. These counter-indications can easily be overcome by developing a stochastic version of the ODE model 3 supporting a discrete number of flocks (see Gillespie 1977). In agreement with the assumptions of the deterministic model 3 , we introduced demographic stochasticity at the flock level only. Because within-farm transmission and prevalence of AI is usually much higher than between-farm transmission (Savill et al. 2006; Truscott et al. 2007), the stochastic processes that favor disease fade out may act primarily at the flock level. Following Durrett and Levin (1994), we assumed that the biological processes in model 3 occur asynchronously. This implies that, given the state of the system at time $t$, the waiting time for the next event to occur is exponentially distributed with a rate given by the sum of the rates of all possible events. In line with these assumptions, we derived the rules for the transition rates listed in Table 1. We then performed two sets of stochastic simulations in order to compare the duration of HPAI outbreaks (1) in the absence and (2) in the presence of LPAI strains. In the first case, the initial conditions for the model variables were set to a single HPAI-infected flock in a totally susceptible population of poultry farms. In the second case, the initial conditions for the model variables were set to a single HPAI-infected flock in a population of poultry farms close to its LPAI-endemic equilibrium, corresponding to: $\left[S\right.$, full-blown infections $\left(I_{H}\right)$, $\left.I_{L}, R_{L}, I_{M}, R_{M}\right]=\left[\bar{S}, 1, \bar{I}_{L}, \bar{R}_{L}, 0,0\right]$, where $\bar{S}, \bar{I}_{L}, \bar{R}_{L}$ are the equilibrium values in model 2 . We then estimated the duration of HPAI outbreak in the presence and in the absence of LPAI virus for different values of the transmission rates $\beta_{H}$ and $\beta_{L}$ by running 10,000 simulations for each combination of the parameters. The stochastic model was thus used to investigate whether the presence of LPAI strains 
Table 1 Rules for the transition rates in the stochastic model

$X=\left(S, I_{H}, I_{L}, R_{L}, I_{M}, R_{M}\right)$; other variables and parameters have the same meaning as in (3)

\begin{tabular}{lll}
\hline Transition rule & Rate & Event \\
\hline$S \rightarrow S+1$ & $c$ & Restocking \\
$X \rightarrow X-1$ & $\mu X$ & Natural death \\
$S, I_{H} \rightarrow S-1, I_{H}+1$ & $\beta_{H} S\left(I_{H}+I_{M}\right)$ & Full-blown HPAI infection \\
$S, I_{L} \rightarrow S-1, I_{L}+1$ & $\beta_{L} S I_{L}$ & LPAI infection \\
$I_{H} \rightarrow I_{H}-1$ & $\alpha_{H} I_{H}$ & Disease-induced death \\
$I_{L}, R_{L} \rightarrow I_{L}-1, R_{L}+1$ & $\delta_{L} I_{L}$ & Loss of LPAI infection \\
$R_{L}, I_{M} \rightarrow R_{L}-1, I_{M}+1$ & $\sigma \beta_{H} R_{L}\left(I_{H}+I_{M}\right)$ & Mild HPAI infection \\
$I_{M}, R_{M} \rightarrow I_{M}-1, R_{M}+1$ & $\delta_{M} I_{M}$ & Loss of mild HPAI infection \\
\hline
\end{tabular}

may significantly affect the time to extinction and persistence of an HPAI strain. Results of the stochastic simulations were then compared to those of the deterministic version of the model.

\section{Parameter values and model analysis}

The parameter values used for numerical simulations are summarized in Table 2. We assumed that the number $K$ of backyard flocks at the disease-free equilibrium is 500 flocks (i.e., the same order of magnitude observed just before the LPAI outbreaks in Northern Italy in 2000-2005 by Busani et al. 2007). Following Iwami et al. (2009), life expectancy of bird stocks on a small-scale farm $(1 / \mu)$ was set to 100 days and thus $\mu=0.274$ years $^{-1}$. Therefore, the restocking rate is $c=\mu K=27.4$ flocks per year. The mean flock infectious period for HPAI in four recent epidemics was estimated by Garske et al. (2007) as 2.5-13 days (mean ca. 7 days), yielding $\alpha_{H}=52.1$ years $^{-1}$. On the other hand, the mean number of weeks during which a flock remained infected with LPAI virus was estimated to be about five yielding $\delta_{L}=10.4$ years $^{-1}$ (Bos et al. 2009). Van der Goot et al. (2003) performed infection experiments with HPAI strains on susceptible chickens and chickens already infected by LPAI strain showing that LPAI provides effective protection against infection and mortality caused by HPAI viruses. In particular, they showed a $100 \%$ survival probability to HPAI infection in hosts already infected by LPAI $\left(\alpha_{M}=0\right)$ and a $40 \%$ reduction in effective transmission in the same hosts $(\sigma=0.4)$. Unfortunately, we are unaware of published studies on flock infectious period for mild influenza infections and so we assumed a flock infectious period similar to that for LPAI because mild influenza infections seem nearly asymptomatic (van der Goot et al. 2003). Therefore, $\delta_{M}$ was set to 10.4 years $^{-1}$.

Despite multiple attempts to assess transmissibility of LPAI and HPAI viruses in domestic poultry, mechanisms of influenza virus transmission are poorly understood (Alexander 2007). To our knowledge, avian influenza transmission has only been studied at the level of bird-tobird contacts under experimental conditions (van der Goot et al. 2003, 2005). However, there is a considerable gap between bird-to-bird transmission with a few animals and the field conditions of within-farm and farm-to-farm disease spread (as highlighted by Bos et al. 2007). Instead, information is available on avian influenza basic reproduction number $\left(\mathfrak{R}_{0}\right)$ - the average number of secondary infections caused by the primary case in a totally susceptible population - which is positively correlated to virus transmission (Anderson and May 1991). If $\mathfrak{R}_{0}>1$, a typical infective gives rise, on average, to more than one secondary infection, leading to an epidemic. On the contrary, if $\Re_{0}<1$, the pathogen cannot establish in the population and eventually goes extinct. Since the ability of the virus to spread is related to the amount of pathogen released by an infected individual, it is possible that relatively little virus is released during the course of HPAI infections because of the
Table 2 Parameter values for model (3)

$y$ years, see the main text for more details

\begin{tabular}{lll}
\hline Parameter & Symbol & Value \\
\hline Restocking rate & $c$ & $137{\text { farm } \times \text { year }^{-1}}^{-1}$ \\
Mortality rate & $\mu$ & $0.274 \mathrm{year}^{-1}$ \\
HPAI induced mortality & $\alpha_{H}$ & $52.1 \mathrm{year}^{-1}$ \\
LPAI recovery rate & $\delta_{L}$ & $10.4 \mathrm{year}^{-1}$ \\
Mild-HPAI induced mortality & $\alpha_{M}$ & 0 \\
Mild-HPAI recovery rate & $\delta_{M}$ & $10.4 \mathrm{year}^{-1}$ \\
Transmission reduction in LPAI recovered & $\sigma$ & 0.4 \\
\hline
\end{tabular}


extremely rapid deaths of infected birds. A poorer infectedto-susceptible transmission is therefore expected in HPAI viruses than in LPAI (Alexander 2007). Estimates for the HPAI basic reproduction number $\mathfrak{R}_{0}(H)$ show high variability in the force of infection, ranging from 0.9 to 2.5 in Bouma et al. (2009), from 1.9 to 2.7 in Ward et al. (2009), and from 1.1 to 3.2 in Garske et al. (2007). The LPAI basic reproduction number $\mathfrak{R}_{0}(L)$ also shows high variability ranging from 2.3 to 3.9 (Marangon et al. 2007). Therefore, in order to overcome the lack of information on and uncertainty in transmission parameters, we performed a sensitivity analysis of infection persistence over a wide range of transmission rates (and thus of $\mathfrak{R}_{0}$ ) of both HPAI and LPAI viruses.

\section{Results}

Deterministic model results

In the specific case of a system of $K$ susceptible backyard farms, the basic reproduction number $\mathfrak{R}_{0}(H)$ for HPAI alone [model 1] can be computed as follows (van den Driessche and Watmough 2002; Iwami et al. 2007):

$\Re_{0}(H)=\frac{\beta_{H} K}{\left(\mu+\alpha_{H}\right)}$

while $\mathfrak{R}_{0}(L)$ for model 2 when LPAI is isolated is:

$\Re_{0}(L)=\frac{\beta_{L} K}{\left(\mu+\delta_{L}\right)}$.

However, as observed in several HPAI outbreaks by Alexander (2000) and Koch and Elbers (2006), LPAI viruses are usually already endemic when a highly pathogenic mutant arises or a backyard farm is infected by an introduced HPAI-infected bird. As a consequence, some portion of the host population has already been infected and recovered from the LPAI virus, thus developing partial resistance to HPAI. Therefore, the best measure to estimate HPAI persistence success in this scenario is the so-called invasion reproduction number $\left.\bar{\Re}_{0}(H)\right|_{L}$, which is defined as the total number of new HPAI infectious cases from a single infectious case in an LPAI-endemic population. The invasion reproduction number is a measure of the relative fitness of one strain when another strain is already established in the population and is considered as a competitive condition, which represents the advantage of the HPAI [LPAI] strain against the LPAI [HPAI] strain. If the invasion reproduction numbers of a strain is less than one, then the strain dies out and only the other strain persists. On the other hand, if both invasion reproduction numbers are greater than one, then both strains can coexist. In the specific case of model 3 , the invasion reproduction number of the HPAI strain when the LPAI infection is at its endemic equilibrium is computed as follows (see Appendix 1 for details):

$\left.\bar{\Re}_{0}(H)\right|_{L}=\frac{\Re_{0}(H)}{\Re_{0}(L)}+\frac{\sigma \beta_{H} \bar{R}_{L}}{\left(\mu+\alpha_{M}+\delta_{M}\right)}$

where, $\mathfrak{R}_{0}(H)$ and $\mathfrak{R}_{0}(L)$ are the single-strain basic reproduction numbers defined in (4) and (5), respectively; $\mathfrak{R}_{0}(H) / \mathfrak{R}_{0}(L)$ is the expected number of full-blown infections $\left(I_{H}\right)$ from a single HPAI infectious case (mild or full-blown) in an LPAI-endemic population; $\sigma \beta_{H} \bar{R}_{L} /\left(\mu+\alpha_{M}+\delta_{M}\right)$ is the expected number of $I_{M}$ from a single HPAI infectious case (mild or full blown) in an LPAI-endemic population; $\bar{R}_{L}=$ $\delta_{L}\left(\Re_{0}(L)-1\right) / \beta_{L}$ is the number of LPAI immune flocks at model 2 endemic equilibrium. Then, we expect a mutant HPAI virus to invade in an LPAI-endemic landscape only if its basic reproduction number exceeds the threshold value $\left.\bar{\Re}_{0}(H)\right|_{L}>1$. Interestingly, according to Eq. 6, an HPAI strain can invade an established LPAI strain when its basic reproduction number is less than one. In fact, by imposing $\left.\bar{\Re}_{0}(H)\right|_{L}>1$ and $\Re_{0}(H)<1$, the parametric condition under which HPAI may establish in the presence of LPAI strains while it would go extinct in a naïve host population can be derived by rearranging Eq. 6 as follows:

$\Re_{0}(M)=\frac{\sigma \beta_{H} K}{\left(\mu+\alpha_{M}+\delta_{M}\right)}>\frac{\left(\mu+\delta_{L}\right)}{\delta_{L}}$.

Therefore, by assuming an LPAI infectious period significantly shorter than the host life expectancy $\left(\delta_{L}>>\mu\right)$, we may expect an unfit HPAI strain [i.e., with $\mathfrak{R}_{0}(H)<1$ ] to spread successfully when the number of secondary mild infections in a population close to its carrying capacity $\mathfrak{R}_{0}(M)$ is larger than one. Moreover, as $\beta_{H} K<\left(\mu+\alpha_{H}\right)$ for $\mathfrak{R}_{0}(H)<1$ [see (4)], we may expect an unfit HPAI strain to spread in a population when its full-blown infectious period $\left(\mu+\alpha_{H}\right)^{-1}$ is appreciably shorter than the duration of a mild infection $\left(\mu+\alpha_{M}+\delta_{M}\right)^{-1}$. Interestingly, Grenfell (2001) showed that the last condition usually holds when mild infections imply reduced host susceptibility (i.e., $\sigma<1$ ) as in model 3 .

In order to assess LPAI persistence or exclusion after a successful HPAI invasion in model 3, we computed the invasion reproduction number for LPAI:

$\left.\bar{\Re}_{0}(L)\right|_{H}=\frac{\Re_{0}(L)}{\Re_{0}(H)}$.

An LPAI strain may persist in a population successfully infected by an HPAI strain only if $\left.\bar{\Re}_{0}(L)\right|_{H}>1$ (i.e., only if LPAI has a larger basic reproduction number than HPAI).

While persistence conditions can be handled analytically, the complete model 3 dynamics are too complex to be 
investigated with analytical tools. Therefore, the remainder of the analyses were carried out through bifurcation methods performed numerically via specialized software implementing continuation techniques, such as LOCBIF (Khibnik et al. 1993) and CONTENT (Kuznetsov 1998).

Figure 2 shows the model 3 bifurcation diagram in the parameter space of the basic reproduction numbers $\mathfrak{R}_{0}(H)$ and $\mathfrak{R}_{0}(L)$ as this summarizes conditions for coexistence and exclusion of the two strains as a function of the other epidemiological parameters entering $\mathfrak{R}_{0}$ (i) computation, such as the transmission rates $\beta_{i}$. The horizontal axis $\left[\mathfrak{R}_{0}\right.$ $(L)=1]$ represents the HPAI dynamics for different values of basic reproduction number in the absence of LPAI viruses as described in model 1. Curve $T_{H}$ represents the threshold for HPAI strain establishment (which corresponds to $\left.\bar{\Re}_{0}(H)\right|_{L}=1$ ) that separates region $\mathrm{A}$ in which the HPAI fails to establish itself from region $\mathrm{C}$ in which the HPAI strain is able to invade the host population converging toward a coexistence equilibrium with the LPAI strain. Similarly, curve $T_{L}$ represents the threshold for LPAI strain establishment (which corresponds to $\left.\bar{\Re}_{0}(L)\right|_{H}=1$ ) that separates region B in which the LPAI fails to establish itself from the coexistence region C. Curve $H$ (Hopf bifurcation) separates the coexistence equilibrium region $\mathrm{C}$ (endemic dynamics) from region $\mathrm{D}$, where model 3 exhibits sustained cycles in abundance of both pathogen strains (epidemic dynamics). The thin lines give the expected cycle lengths in regions where cycles occur (in years).

As highlighted in (7), Fig. 2 shows that, when LPAI in endemic the host population (i.e., $\mathfrak{R}_{0}(H) \mathfrak{R}_{0}(L)>1$ ), HPAI outbreaks may occur even when $\mathfrak{R}_{0}(H)<1$. Furthermore, such outbreaks may exhibit inter-annual periodicity with large peaks of infection every 5-10 years (dark gray region).

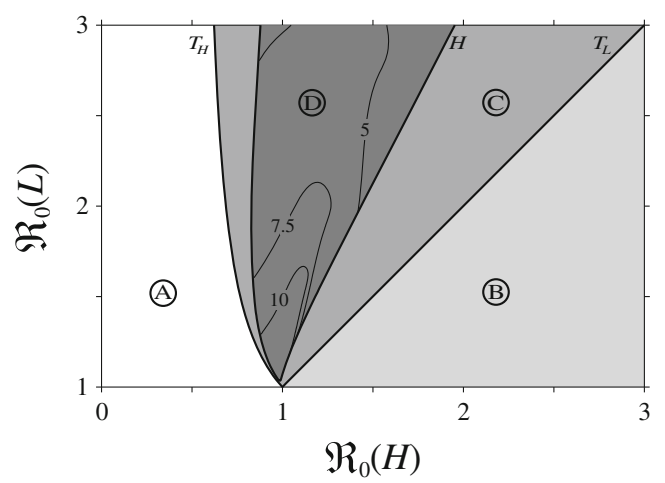

Fig. 2 Model 3 bifurcation diagram in the $\left(\Re_{0}(H), \Re_{0}(L)\right)$ space. All parameters are held constant apart from the transmission rates $\beta_{H}$ and $\beta_{L} . T_{H}$ : threshold for HPAI strain establishment; $T_{L}$ : threshold for LPAI strain establishment; $H$ : Hopf bifurcation. In region (A) only LPAI persists, (B) only HPAI persists, (C) endemic coexistence, (D) epidemic coexistence. The thin lines give the expected cycle lengths in regions where cycles occur. Other parameters as in Table 2
Stochastic model results

Stochastic models are useful for investigating pathogen extinction (self-limiting epidemic) or persistence (long-term epidemic) in the host population. We assessed whether the presence of LPAI strains affects the time to extinction for an unfit HPAI strain with $\mathfrak{R}_{0}(H) \leq 1$.

The gray region in Fig. 3 shows the area in the parameter space $\left[\mathfrak{R}_{0}(H), \mathfrak{R}_{0}(L)\right]$ where HPAI epidemics in the presence of LPAI are longer than HPAI epidemics in an initially naïve population at its carrying capacity $K=c / \mu$. Ten thousand replicates were run for each set of parameter values. For each replicate, simulations were run until HPAIinfected individuals no longer existed in the population. Gray areas represent the combination of $\left[\mathfrak{R}_{0}(H), \mathfrak{R}_{0}(L)\right]$ values for which the average duration of HPAI epidemics in the presence of LPAI is $50 \%$ (light gray) and $100 \%$ (dark gray) longer, respectively, than HPAI epidemics in an initially naïve population (i.e., a population not previously exposed to LPAI viruses). The black curve represents the invasion threshold derived through the deterministic model 3.

Although results of the differential equation model are dependent on the treatment of individuals as continuous entities, analogous behaviors are observed in the stochastic system with discrete individuals when a pathogen is introduced into an LPAI-endemic population. The invasion of an HPAI strain into an LPAI-endemic population often leads to a longer epidemic than an introduction into a susceptible population.

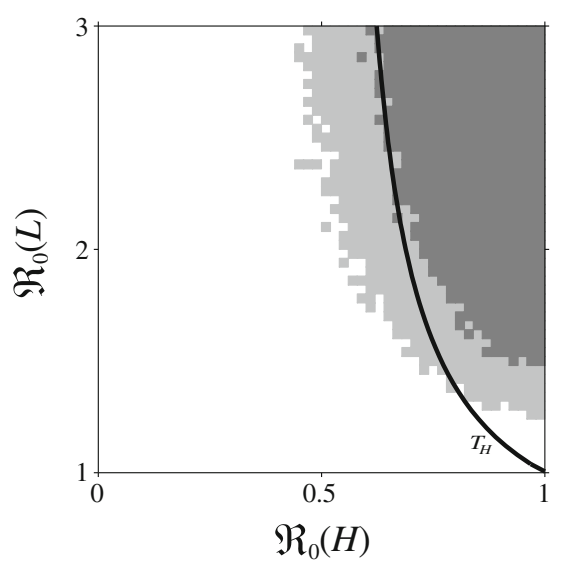

Fig. 3 Light and dark gray regions identify the combination of $\left[\Re_{0}(H)\right.$, $\mathfrak{R}_{0}(L)$ ] values where the average duration of HPAI epidemics in the presence of LPAI are $50 \%$ and $100 \%$ longer than the duration of HPAI epidemics in a naïve population, respectively. Simulations of infectious dynamics were replicated 10,000 times for each set of parameter values $\left[\mathfrak{R}_{0}(H), \mathfrak{R}_{0}(L)\right]$. All model parameters are held constant apart from the transmission rates $\beta_{H}$ and $\beta_{L}$ as in Fig. 2. Black curve $\left(T_{H}\right)$ represents the invasion threshold computed as in model 3. The farm population size is $c / \mu=500$. Other parameters as in Table 2 


\section{Discussion}

We explored the epidemiological dynamics and persistence of high pathogenicity avian influenza and its interaction with low pathogenicity avian influenza. The analysis was carried out using two techniques: deterministic dynamics and stochastic simulations. These two approaches provide complementary predictions on the fate of the two strains. They both show that the presence of low pathogenicity strains plays a crucial role in determining the fate of high pathogenicity avian influen$\mathrm{za}$, enhancing both its probability of persistence and the duration of its epidemics. In fact, as hosts already infected by LPAI strains may experience milder symptoms of HPAI, the presence of LPAI strains increases the disease infectious period [see (7)]; this allows the persistence of HPAI strains even when their basic reproduction number in naïve populations is lower than one.

The possibility that HPAI exposure may yield a mild infection due to the partial cross-immunity conferred by LPAI has been well documented in laboratory experiments with caged chickens (van der Goot et al. 2003, 2005): these studies show that LPAI-positive birds survived to a HPAI infection. In the field, low mortality was reported during a HPAI outbreak in Chile in 2002 (Verdugo et al. 2009).

We investigated avian influenza dynamics only among domestic birds. Obviously, the effect of wild bird migration is also considered an important risk factor for disease spread (Normile 2005). However, some H5N1 cases in poultry have been reported in countries in which infections are not associated with migratory bird movements (Kilpatrick et al. 2006). Therefore, the exclusion of wild birds from our analysis does not invalidate model results.

The ecological literature contains numerous examples of cross-protection mechanisms that favor strain coexistence (Castillo-Chavez et al. 1989; Andreasen et al. 1997). Moreover, other theoretical work shows that, in specific conditions, cross-immunity may favor strain coexistence even in the case of unfit invading mutants (i.e., with basic reproduction number lower than one) as in model 3 (White et al. 1998; Nuño et al. 2007). White et al. (1998) showed that in the presence of cross-immunity, persistence of unfit, nonlethal strains is possible when transmission rates of secondary infections are greater than transmission rates of primary infections. Nuño et al. (2007) showed that when infected individuals are isolated, cross-immunity may favor the coexistence of human influenza strains even in the case of unfit invading mutants. Here, we showed that in the case of avian influenza on poultry farms where previous conditions do not apply, highly pathogenic unfit strains can also invade and establish in a partially immune population.
Cross-immunity may generate persistence of unfit strains (with $R_{0}<1$ ) through the emergence of so-called backward bifurcations (Greenhalgh et al. 2000). Typically, infection models show a smooth stability change (i.e., a smooth increase in prevalence) at the disease persistence threshold (called forward bifurcation) in which an endemic equilibrium with few infected individual arises. However, in the presence of cross-immunity, infection models may exhibit backward bifurcations in which an endemic equilibrium coexists with the disease-free equilibrium for transmission rates lower than threshold persistence, $R_{0}=1$ (Greenhalgh et al. 2000). In some cases, uncontrolled epidemic systems that exhibit forward bifurcations may produce backward bifurcations after the implementation of vaccine policies introducing partial immunity into the host population (Kribs-Zaleta and Velasco-Hernandez 2000; Arino et al. 2003).

Our analysis shows that while each strain would go toward a stable endemic equilibrium when alone (or, in the case of HPAI virus, would possibly fade out), the interacting dynamics of LPAI- and HPAI-infected flocks may give rise to multiyear periodicity with high epidemic peaks and low prevalence, inter-epidemic periods ranging from few to several years. While it is unlikely that the HPAI infection would persist with boom-and-bust dynamics for several years in a poultry farm system (due to the limited number of exposed farms), the presence of large oscillations in the epidemiological dynamics may imply a larger epidemic wave during disease invasion. Similar patterns of dumped oscillations or limit cycles with high epidemic picks and deep troughs have been observed in other multistrain models with cross-immunity including whooping cough (Restif and Grenfell 2006), dengue (Ferguson et al. 1999), malaria (Gupta et al. 1994), and human influenza (Ferguson et al. 2003; Casagrandi et al. 2006; Koelle et al. 2006). Typical multistrain models consider two or more strains with similar phenotypic traits (infectious period and transmission rate) and different degrees of crossprotection. For instance, Andreasen et al. (1997) showed that sustained oscillations may occur in simple human influenza models of three or more strains. In the present case of avian influenza, sustained oscillations arose through Hopf bifurcations for two strains with remarkable differences in pathogenicity and some level of cross-immunity. The analysis of our stochastic LPAI-HPAI model showed that the invasion of an HPAI strain into an LPAI-endemic population may lead to longer epidemics than in the case of a totally susceptible population; this effect is greater for LPAI infection with larger basic reproduction number (see Fig. 3). The presence of partially immune individuals previously exposed to LPAI resulted in a lower HPAI force of infection; thus, instead of generating boom-and-bust epidemics leading to fast extinction, HPAI generates a 
smaller epidemic with a less dramatic depletion of individuals. This may allow the pathogen to persist in the population for longer periods of time. While we do not rule out other mechanisms fostering HPAI persistence - such as the mutation of HPAI viruses toward less virulent strains or an increase in connectivity and size of the susceptible populations - we note that our predictions are consistent with recent reports that HPAI strains have become endemic in China (Alexander 2007).

Grenfell (2001) obtained comparable results with an SIR framework describing the so-called invasion-persistence trade-off, while King et al. (2009) generalized their finding in a metapopulation framework. They suggested that virulent strains with short infectious period and high transmission rate (as in the full-blown HPAI infection) display competitive advantages spreading faster in the host population than strains with longer infectious period and lower instantaneous transmission rate (as in the mild HPAI infection). On the other hand, more transmissible strains suffer greater risk of fade out exhibiting epidemic dynamics with larger boom-and-bust fluctuations. Thus, invasion advantages for more virulent strains come at the cost of diminished persistence probability (Grenfell 2001). Grenfell et al. found that the invasion-persistence trade-off may produce selective pressures favoring the persistence of strains with intermediate virulence. In light of this, we believe that a specific function linking reduction of susceptibility and duration of infection (that is, the additional death rate) could be introduced into model 3 to analyze the evolutionary proprieties of the system following game theoretic (Metz et al. 1996) or quantitative genetics (Day and Proulx 2004) approaches. This issue will be object of further investigations.

Savill et al. (2006) demonstrated a similar phenomenon by modeling the H5N1 avian influenza introduction into partially vaccinated poultry populations. By using a detailed stochastic model, the authors showed that partial vaccination could promote undetected pathogen persistence facilitating the spread of avian influenza to neighboring farms. In the same way, Pulliam et al. (2007a) predicted, with deterministic and stochastic analyses, longer epidemic events when a human pathogen (such as measles and rubella) is introduced or reintroduced into a partially immune population. They called this phenomenon epidemic enhancement. Some evidence for this effect has also been found in the field. Analyzing livestock production data following the Nipah encephalitis outbreak on Malaysian pig farms (1998-1999), Pulliam et al. (2007b) found that previous Nipah virus introduction produced partial immunity in pigs, which led to a more widespread and persistent epidemic than would have been observed if the pig population had been entirely naïve (Pulliam et al. 2007b).
An alternative mechanism proposed for avian influenza persistence, especially in wild waterfowl, is transmission from propagules (i.e., viral particles) that persist for long periods of time outside of the host in the environment ("environmental transmission"). By using deterministic and stochastic models of avian influenza in waterfowl, Rohani et al. (2009) showed that a very low rate of environmental transmission (relative to rates of direct transmission) may dramatically increase the probability of pathogen persistence providing a parsimonious explanation for the observed epidemic pattern of avian influenza in the wild. Therefore, we may expect similar effects in domestic birds, where the virus spreads through direct bird-to-bird contact as well as through indirect contact via contaminated feed, equipment, water, and personnel. The potential presence of alternative transmission routes in domestic avian influenza may favor the persistence of strains with low bird-to-bird basic reproduction numbers (Roche et al. 2005).

The interacting dynamics of HPAI and LPAI investigated in the present paper may suggest an alternative mechanism to explain HPAI circulation in the wildlife. LPAI viruses have been isolated from at least 105 wild bird species of 26 different families (Olsen et al. 2006), but waterfowl birds, such as the Anseriformes (particularly ducks, geese, and swans) and Charadriiformes (particularly gulls, terns, and waders), constitute the major natural LPAI virus reservoir. In these species, LPAI viruses may attain a significant prevalence and generally cause no evident clinical signs. HPAI infections were not detected in the wild before 2002 but in the past decade, HPAI H5N1 viruses have been reported in more than 100 species of wild birds (USGS 2008) usually found dead or diseased. A number of freeliving wildfowl species, including mallard ducks and barheaded geese, have been shown to asymptomatically shed a large number of viral propagules for several days without exhibiting any apparent clinical signs or prior to the onset of illness. Despite such great inter- and intraspecific variability in the susceptibility to, and virulence of, HPAI viruses (reviewed by Pantin-Jackwood and Swayne 2009), HPAI outbreaks are believed to be self-limiting in wildlife and to be triggered by spillover from gallinaceous poultry (Webster et al. 2007). Outbreaks of HPAI virus at Qinghai Lake in 2005-2006 and Poyang Lake in 2006 demonstrate that dense congregation of wild birds at feeding or reproductive sites may counteract barriers to HPAI transmission (Takekawa et al. 2010). Given recurrent outbreaks in wildlife in the past 5 years, it has been questioned whether highly pathogenic $\mathrm{H} 5 \mathrm{~N} 1$ viruses are becoming endemic in wild waterfowl (Webster et al. 2007). In this respect, a crucial issue that remains unaddressed is the possible effect of cocirculating influenza viruses in wild birds on the pathogenicity of HPAI viruses (Webster et al. 
2006). We ran other simulations by extending our flockbased model to describe the epidemiological dynamics of a self-limiting, free-ranging wild bird population characterized by logistic growth when disease free. The results of this additional analysis (not reported here) show that the main conclusions of the present work-i.e., (a) persistence of an otherwise unfit HPAI virus when LPAI is endemic and (b) potential for inter-annual periodicity when the two strains are both present-also hold true for the dynamics of avian influenza in wild bird populations. As a consequence, partial cross-protection provided by previous exposure to LPAI could theoretically allow HPAI to become entrenched in wild waterfowl cycles, without necessarily requiring repeated spill-over from domestic poultry to explain interannual boom-and-bust dynamics of HPAI in wildlife.

Another important aspect in the spread of HPAI is that an increase in life expectancy of an HPAI-infected bird previously exposed to LPAI strains may allow migrant birds to fly long distances before the onset of the disease. For instance, during the asymptomatic period, bar-headed geese can potentially fly over $500 \mathrm{~km}$ (Gaidet et al. 2010) suggesting that they could serve as long-distance carriers spreading HPAI between distant populations and/or species. Periodic aggregation around common feeding sites for overwintering or during migration may also provide opportunities for HPAI to infect a large number of species and individuals. As migration and aggregation usually occur in predictable temporal patterns, it is therefore crucial that seasonality in contact rates be accounted for in future modeling effort of wild bird avian influenza. Several theoretical and empirical works have shown that the explicit inclusion of periodic forcing in models of wildlife disease may important for accurately describing epidemics of wildlife in strongly seasonal environments (Altizer et al. 2006; Bolzoni et al. 2008). Reproduction, for instance, is another seasonal process that could play a crucial role in the dynamics of avian influenza in the wild. In fact, the reproductive pulse of immunologically naïve young birds in breeding sites can potentially cause higher HPAI prevalence and bird losses (Hénaux et al. 2010).

Our analysis was primarily applied to a homogeneous population of flocks (or wild birds) under the constraints of limited availability of data on contact rates. While this, in itself, is a valuable exercise, the ultimate goal will be to apply this framework to a heterogeneous system of poultry farms (or a metapopulation of wild birds) where flocks (or wild bird populations) differ in species, size, and connectivity. This effort would resemble that of Truscott et al. (2007) who described the dynamics of single-strain HPAI epidemics in the network of poultry farms in Great Britain. Several other important heterogeneities were not included in our modeling exercise, such as more detailed structure of contact processes, species-specific susceptibility, withinflock dynamics, seasonality in transmission rates, and strain evolution. For example, the dynamics of influenza evolution are intrinsically transient in the sense that new types are continuously introduced while old types die out. A multistrain approach, such as that developed by Gog and Grenfell (2002), would help to provide a more realistic description of epidemiological dynamics and of the role of cross-protection.

We deliberately kept our model as simple as possible so as to analyze specifically the role of cross-immunity acquired through infection with LPAI viruses in the persistence of an unfit HPAI virus that would otherwise rapidly fade out. Whether through mutation/reassortment or through cross-protection, HPAI viruses seem to need LPAI infection to invade and persist in a system of poultry farms.

Webster et al. (2006) observed that clinical signs of infection with highly pathogenic viruses may be masked by cross-protection by LPAI strains, a fact largely overlooked in the analysis of epidemiological data on avian influenza, both in domestic poultry and in wild bird species. While further field work and laboratory research is required to cast light on this question, our theoretical work shows that this is indeed a potential mechanisms for the persistence of highly pathogenic viruses of avian influenza and that, under specific circumstances, such cross-protection may trigger complex dynamics exhibiting multiyear periodicity.

Acknowledgments The authors are very thankful to Vittorio Guberti for his valuable suggestions on an early version of the model, to Mercedes Pascual for her priceless and constructive remarks that helped us to improve the work, and to Chelsea Wood for her insightful comments on the final version of the paper. This work was partially supported by the Interlink Program by the Italian Minister of Research (project II04CE49G8).

Open Access This article is distributed under the terms of the Creative Commons Attribution Noncommercial License which permits any noncommercial use, distribution, and reproduction in any medium, provided the original author(s) and source are credited.

\section{Appendix 1: Computation of the HPAI invasion reproduction number}

We define the invasion reproduction number of the HPAI as the spectral radius of the "next generation" matrix $\mathrm{FV}^{-1}$ (Diekmann et al. 1990), that is: $\left.\bar{\Re}_{0}(H)\right|_{L}=\rho\left(F V^{-1}\right)$, where $F$ and $V$ are defined as a Jacobian matrix of the rate of appearance of new HPAI infections $(f)$ and the Jacobian matrix of other rates of transfer $(v)$ calculated for infected compartments (i.e., $\dot{I}_{H}$ and $\dot{I}_{M}$ ) in the LPAI-endemic equilibrium $\left[S, I_{H}, I_{L}, R_{L}, I_{M}, R_{M}\right]=\left[\bar{S}, 0, \bar{I}_{L}, \bar{R}_{L}, 0,0\right]$, where 
$\bar{S}, \bar{I}_{L}, \bar{R}_{L}$ are the equilibrium values in model 2.

$f=\left[\begin{array}{l}\beta_{H} S I_{T} \\ \sigma \beta_{H} \bar{R}_{L} I_{T}\end{array}\right]$ and $v=\left[\begin{array}{l}\left(\mu+\alpha_{H}\right) I_{H} \\ \left(\mu+\alpha_{M}+\delta_{M}\right) I_{M}\end{array}\right]$,

where, $I_{T}=I_{H}+I_{M}$. The Jacobian matrices at the LPAIendemic equilibrium are as follows:

$F=\left[\begin{array}{ll}\beta_{H} \bar{S} & \beta_{H} \bar{S} \\ \sigma \beta_{H} \bar{R}_{L} & \sigma \beta_{H} \bar{R}_{L}\end{array}\right]$ and $V=\left[\begin{array}{cc}\mu+\alpha_{H} & 0 \\ 0 & \mu+\alpha_{M}+\delta_{M}\end{array}\right]$

Then, the next generation matrix $F V^{-1}$ is:

$$
\begin{aligned}
F V^{-1}= & \frac{1}{\left(\mu+\alpha_{H}\right)\left(\mu+\alpha_{M}+\delta_{M}\right)} \\
& \times\left[\begin{array}{ll}
\beta_{H} \bar{S}\left(\mu+\alpha_{M}+\delta_{M}\right) & \beta_{H} \bar{S}\left(\mu+\alpha_{H}\right) \\
\sigma \beta_{H} \bar{R}_{L}\left(\mu+\alpha_{M}+\delta_{M}\right) & \sigma \beta_{H} \bar{R}_{L}\left(\mu+\alpha_{H}\right)
\end{array}\right]
\end{aligned}
$$

and its spectral radius is:

$$
\begin{aligned}
\left.\bar{\Re}_{0}(H)\right|_{L} & =\rho\left(F V^{-1}\right)=\frac{\beta_{H} \bar{S}}{\left(\mu+\alpha_{H}\right)}+\frac{\sigma \beta_{H} \bar{R}_{L}}{\left(\mu+\alpha_{M}+\delta_{M}\right)} \\
& =\frac{\Re_{0}(H)}{\Re_{0}(L)}+\frac{\sigma \beta_{H} \bar{R}_{L}}{\left(\mu+\alpha_{M}+\delta_{M}\right)} .
\end{aligned}
$$

\section{References}

Abu-Raddad LJ, Ferguson NM (2004) The impact of cross-immunity, mutation and stochastic extinction on pathogen diversity. Proc R Soc B 271:2431-2438

Alexander DJ (2000) A review of avian influenza in different bird species. Vet Microbiol 74:3-13

Alexander DJ (2007) An overview of the epidemiology of avian influenza. Vaccine 25:5637-5644

Altizer S, Dobson AP, Hosseini P, Hudson P, Pascual M, Rohani P (2006) Seasonality and the dynamics of infectious diseases. Ecol Lett 9:467-484

Anderson RM, May RM (1991) Infectious disease of humans: dynamics and control. Oxford University Press, Oxford

Andreasen V, Lin J, Levin SA (1997) The dynamics of cocirculating influenza strains conferring partial cross-immunity. J Math Biol $35: 825-842$

Arino J, McCluskey CC, Van den Driessche P (2003) Global results for an epidemic model with vaccination that exhibits backward bifurcation. SIAM J Appl Math 64:260-276

Banks J, Speidel EC, McCauley JW, Alexander DJ (2000) Phylogenetic analysis of $\mathrm{H} 7$ haemagglutinin subtype influenza A viruses. Arch Virol 145:1047-1058

Bavinck V, Bouma A, van Boven M, Bos MEH, Stassen E, Stegeman JA (2009) The role of backyard poultry flocks in the epidemic of highly pathogenic avian influenza virus (H7N7) in the Netherlands in 2003. Prev Vet Med 88:247254

Bean WJ, Kawaoka Y, Wood JM, Pearson JE, Webster RG (1985) Characterization of virulent and avirulent A/Chicken/Pennsylvania/
83 influenza A viruses: potential role of defective interfering RNAs in nature. J Virol 54:151-160

Bicknell KB, Wilen JE, Howitt RE (1999) Public policy and private incentives for livestock disease control. Aust J Agric Resour Econ 43:501-521

Bolzoni L, Dobson AP, Gatto M, De Leo G (2008) Allometric scaling and seasonality in the epidemics of wildlife diseases. Am Nat 172:818-828

Bos MEH, van Boven M, Nielen M, Bouma A, Elbers ARW, Nodelijk G, Koch G, Stegeman A, de Jong MCM (2007) Estimating the day of highly pathogenic avian influenza (H7N7) virus introduction into a poultry flock based on mortality data. Vet Res 38:493504

Bos MEH, Busani L, Toson M, Klinkenberg D, Stegeman A, Marangon S Nielen M (2009) Impact of vaccination on between-farm transmission of low pathogenicity avian influenza virus. Meeting of the Society for Veterinary Epidemiology and Preventive Medicine, London, UK. 1st-3rd April 2009

Bouma A, Claassen I, Natih K, Klinkenberg D, Donnelly CA, Koch G, van Boven M (2009) Estimation of transmission parameters of H5N1 avian influenza virus in chickens. PLoS Pathog 5(1): e1000281

Bowes VA, Ritchie SJ, Byrne S, Sojonky K, Bidulka JJ, Robinson JH (2004) Virus characterization, clinical presentation, and pathology associated with H7N3 avian influenza in British Columbia broiler breeder chickens in 2004. Avian Dis 48:928-934

Brown JD, Swayne DE, Cooper RJ, Burns RE, Stallknecht DE (2007) Persistence of $\mathrm{H} 5$ and $\mathrm{H} 7$ avian influenza viruses in water. Avian Dis 51:285-289

Busani L, Dalla Pozza M, Bonfanti L et al (2007) Intervention strategies for low-pathogenic avian influenza control in Italy. Avian Dis 51:470-473

Capua I, Mutinelli F, Marangon S, Alexander DJ (2000) H7N1 avian influenza in Italy (1999 to 2000) in intensively reared chickens and turkeys. Avian Pathol 29:537-543

Casagrandi R, Bolzoni L, Levin SA, Andreasen V (2006) The SIRC model and influenza A. Math Biosci 200:152-159

Castillo-Chavez C, Hethcote HW, Andreasen V, Levin SA, Liu W (1989) Epidemiological models with age structure, proportionate mixing, and cross-immunity. J Math Biol 27:233-258

Chen H, Deng G, Li Z et al (2004) The evolution of H5N1 influenza viruses in ducks in southern China. Proc Natl Acad Sci USA 101:10452-10457

Day T, Proulx SR (2004) A general theory for the evolutionary dynamics of virulence. Am Nat 163:E40-E62

Diekmann O, Heesterbeek JAP, Metz JAJ (1990) On the definition and the computation of the basic reproduction ratio $R_{0}$ in models for infectious diseases in heterogeneous populations. J Math Biol 28:365-382

Durrett R, Levin SA (1994) Stochastic spatial models: a user's guide to ecological applications. Philos Trans Roy Soc B 343:329-350

Elbakidze L (2008) Modeling of avian influenza mitigation policies within the backyard segment of poultry sector. J Agric Resour Econ 33:195-211

Elbers ARW, Fabri THF, Vries TS, De Wit JJ, Pijpers A, Koch G (2004) The highly pathogenic avian influenza A (H7N7) epidemic in the Netherlands in 2003-lessons learned from the first five outbreaks. Avian Dis 48:691-705

Fereidouni SR, Starick E, Beer M, Wilking H, Kalthoff D, Grund C, Hauslaigner R, Breithaupt A, Lange E, Harder TC (2009) Highly pathogenic avian influenza virus infection of mallards with homo- and heterosubtypic immunity induced by low pathogenic avian influenza viruses. PLoS ONE 4:e6706

Ferguson N, Anderson RM, Gupta S (1999) The effect of antibodydependent enhancement on the transmission dynamics and 
persistence of multiple-strains pathogens. Proc Natl Acad Sci USA 96:790-794

Ferguson N, Galvani AP, Bush RM (2003) Ecological and immunological determinants of influenza evolution. Nature 422:428-433

Gaidet N, Cappelle J, Takekawa JY, Prosser D, Iverson S, Douglas DC, Perry WM, Mundkur T, Newman SH (2010) Potential spread of highly pathogenic avian influenza $\mathrm{H} 5 \mathrm{~N} 1$ by wildfowl: dispersal ranges and rates determined from large-scale satellite telemetry. J Appl Ecol 47:1147-1157

Garcia M, Crawford JM, Latimer JW, Rivera-Cruz E, Perdue ML (1996) Heterogeneity in the haemagglutinin gene and emergence of the highly pathogenic phenotype among recent H5N2 avian influenza viruses from Mexico. J Gen Virol 77:1493-1504

Garnett WH (1987) Status of avian influenza in poultry: 19811986. In: Proceedings of the second International Symposium on Avian Influenza. University of Wisconsin, Madison, USA. pp. $61-66$

Garske T, Clarke P, Ghani AC (2007) The transmissibility of highly pathogenic avian influenza in commercial poultry in industrialised countries. PLoS ONE 2(4):e349

Gillespie DT (1977) Exact stochastic simulation of coupled chemical reactions. J Phys Chem 81:2340-2361

Gog JR, Grenfell BT (2002) Dynamics and selection of many-strain pathogens. Proc Natl Acad Sci USA 99:17209-17214

Greenhalgh D, Diekmann O, de Jong MCM (2000) Subcritical endemic steady states in mathematical models for animal infections with incomplete immunity. Math Biosci 165:1-25

Grenfell BT (2001) Dynamics and epidemiological impact of microparasites. In: Smith GL, Irving WL, McCauley JW, Rowlands DJ (eds) New challenges to health: the threat of virus infection. Cambridge University Press, Cambridge, pp 33-52

Gupta S, Swinton J, Anderson RM (1994) Theoretical studies of the effects of heterogeneity in the parasite population on the transmission dynamics of malaria. Proc R Soc B 256:231238

Hénaux V, Samuel MD, Bunck CM (2010) Model-based evaluation of highly and low pathogenic avian influenza dynamics in wild birds. PLoS ONE 5(6):e10997

Henning KA, Henning J, Morton J, Thanh Long N, Truc Ha N, Meers J (2009) Farm- and flock-level risk factors associated with highly pathogenic avian influenza outbreaks on small holder duck and chicken farms in the Mekong Delta of Viet Nam. Prev Vet Med 91:179-188

Iwami S, Takeuchi Y, Liu X (2007) Avian-human influenza epidemic model. Math Biosci 207:1-25

Iwami S, Takeuchi Y, Liu X (2009) Avian flu pandemic: can we prevent it? J Theor Biol 257:181-190

Kalthoff D, Breithaupt A, Teifke JP, Globig A, Harder T, Mettenleiter TC, Beer M (2008) Highly pathogenic avian influenza virus (H5N1) in experimentally infected adult mute swans. Emerg Infect Dis 14:1267-1270

Keeling MJ, Woolhouse MEJ, May RM, Davies G, Grenfell BT (2003) Modelling vaccination strategies against foot-and-mouth disease. Nature 421:136-142

Khibnik AI, Kuznetsov YA, Levitin VV, Nikolaev EV (1993) Continuation techniques and interactive software for bifurcation analysis of ODEs and iterated maps. Phys D 62:360371

Kilpatrick AM, Chmura AA, Gibbons DW, Fleischer RC, Marra PP, Daszak P (2006) Predicting the global spread of H5N1 avian influenza. Proc Natl Acad Sci USA 103:19368-19373

King AA, Shrestha S, Harvill ET, Bjørnstad ON (2009) Evolution of acute infections and the invasion-persistence trade-off. Am Nat $173: 446-455$
Kirupaharan N, Allen LJS (2004) Coexistence of multiple pathogen strains in stochastic epidemic models with density-dependent mortality. Bull Math Biol 66:841-864

Koch G, Elbers ARW (2006) Outdoor ranging of poultry: a major risk factor for the introduction and development of highpathogenicity avian influenza. NJAS-Wagen J Life Sci 54:179194

Koelle K, Cobey S, Grenfell B, Pascual M (2006) Epochal evolution shapes the phylodynamics of interpandemic influenza A (H3N2) in humans. Science 314:1898-1903

Kribs-Zaleta CM, Velasco-Hernandez JX (2000) A simple vaccination model with multiple endemic states. Math Biosci 164:183-201

Kuznetsov Y (1998) CONTENT - integrated environment for analysis of dynamical systems. Tutorial. Rapport de Recherche UPMA98-224, Ecole Normal Superieure de Lyon. Lyon, France

Li KS, Guan Y, Wang J et al (2004) Genesis of a highly pathogenic and potentially pandemic $\mathrm{H} 5 \mathrm{~N} 1$ influenza virus in eastern Asia. Nature 430:209-213

Mannelli A, Busani L, Toson M, Bertolini S, Marangon S (2007) Transmission parameters of highly pathogenic avian influenza (H7N1) among industrial poultry farms in northern Italy in 1999 2000. Prev Vet Med 81:318-322

Marangon S, Busani L, Capua I (2007) Practicalities of the implementation of a vaccination campaign for avian influenza. Avian Dis 51:297-303

Metz JAJ, Geritz SAH, Meszena G, Jacobs FJA, van Heerwaarden JS (1996) Adaptive dynamics, a geometrical study of the consequences of nearly faithful reproduction. In: van Strien S, Lunel SV (eds) Stochastic and spatial structures of dynamical systems. North-Holland, Amsterdam, pp 183-231

Mulatti P, Bos MEH, Busani L, Nielen M, Marangon S (2010) Evaluation of interventions and vaccination strategies for low pathogenicity avian influenza: spatial and space-time analyses and quantification of the spread of infection. Epidemil Infect 138:813-824

Naeem K (1998) The avian influenza H7N3 outbreak in South Central Asia. In: Proceedings of the 4th International Symposium on Avian Influenza, Athens, Georgia. US Animal Health Association, pp. 31-35

Normile D (2005) Are wild birds to blame? Science 310:426-428

Nuño M, Chowell G, Wang X, Castillo-Chavez C (2007) On the role of cross-immunity and vaccines on the survival of less fit flustrains. Theor Pop Biol 71:20-29

Olsen B, Munster VJ, Wallensten A, Waldenström J, Osterhaus AD, Fouchier RA (2006) Global patterns of influenza A virus in wild birds. Science 312:384-388

Pantin-Jackwood MJ, Swayne DE (2009) Pathogenesis and pathobiology of avian influenza virus infection in birds. Rev Sci Tech Off Int Epiz 28:113-136

Pelzel AA, McCluskey BJ, Scott EA (2006) Review of the high pathogenic avian influenza outbreak in Texas, 2004. J Am Vet Med Assoc 228:1869-1875

Poetri ON, Bouma A, Murtini S, Claassen I, Koch G, Soejoedono RD, Stegeman JA, van Boven M (2009) An inactivated H5N2 vaccine reduces transmission of highly pathogenic $\mathrm{H} 5 \mathrm{~N} 1$ avian influenza virus among native chickens. Vaccine 27:2864-2869

Pulliam JRC, Dushoff JG, Levin SA, Dobson AP (2007a) Epidemic enhancement in partially immune populations. PLOS ONE 2: e165

Pulliam JRC, Dushoff J, Field HE, Epstein JH, HERG, Dobson AP, Daszak P (2007b) Understanding Nipah virus emergence in Peninsular Malaysia: the role of epidemic enhancement in domestic pig population. ASTMH: Philadelphia, USA

Restif O, Grenfell BT (2006) Integrating life history and crossimmunity into the evolutionary dynamics of pathogens. Proc R Soc B 273:409-416 
Restif O, Grenfell BT (2007) Vaccination and the dynamics of immune evasion. J R Soc Interface 4:143-153

Roche B, Lebarbenchon C, Gauthier-Clerc M, Chang CM, Thomas F, Renaud F, van der Werf S, Guégan JF (2005) Water-borne transmission drives avian influenza dynamics in wild birds: the case of the 2005-2006 epidemics in the Camargue area. Infect Genet Evol 9(5):800-805

Rohani P, Breban R, Stallknecht DE, Drake JM (2009) Environmental transmission of low pathogenicity avian influenza viruses and its implications for pathogen invasion. Proc Natl Acad Sci USA 106:10365-10369

Rojas H, Moreira R, Avalos P, Capua I, Marangon S (2002) Avian influenza in poultry in Chile. Vet Rec 151:188

Savill NJ, St-Rose SG, Keeling MJ, Woolhouse MEJ (2006) Silent spread of $\mathrm{H} 5 \mathrm{~N} 1$ in vaccinated poultry. Nature 442:757

Stallknecht DE, Brown JD (2008) Ecology of avian influenza in wild birds. In: Swayne DE (ed) Avian influenza. Blackwell, Iowa, pp 43-58

Stegeman A, Bouma A, Elbers AR et al (2004) Avian influenza A virus (H7N7) epidemic in The Netherlands in 2003: course of the epidemic and effectiveness of control measures. J Infect Dis 190:2088-2095

Stollenwerk N, Maiden MCJ, Jansen VAA (2004) Diversity in pathogenicity can cause outbreaks of meningococcal disease. Proc Natl Acad Sci USA 101:10229-10234

Swayne DE, Halvorson DA (2003) Influenza. In: Saif YM, Barnes HJ, Glisson JR, Fadly AM, McDougald LR, Swayne DE (eds) Diseases of poultry, 11 th edn. Iowa State Press, Ames, pp 135-160

Takekawa JY, Prosser DJ, Newman SH, Muzaffar SB, Hill NJ, Yan B, Xiao X, Lei F, Li T, Schwarzbach SE, Howell JA (2010) Victims and vectors: highly pathogenic avian influenza $\mathrm{H} 5 \mathrm{~N} 1$ and the ecology of wild birds. Avian Biol Res 3(2):1-23

Tiensin T, Nielen M, Vernooij $\mathrm{H}$ et al (2007) Transmission of the highly pathogenic avian influenza virus H5N1 within flocks during the 2004 epidemic in Thailand. J Infect Dis 196:1679-1684

Tildesley MJ, Savill NJ, Shaw DJ, Deardon R, Brooks SP, Woolhouse MEJ, Grenfell BT, Keeling MJ (2006) Optimal reactive vaccination strategies for a foot-and-mouth outbreak in the UK. Nature 440:83-86
Truscott J, Garske T, Chis-Ster I, Guitian J, Pfeiffer D et al (2007) Control of a highly pathogenic H5N1 avian influenza outbreak in the GB poultry flock. Proc R Soc B 274:2287-2295

USGS (2008) List of species affected by H5N1 (avian influenza). US Geological Survey. http://www.nwhc.usgs.gov/disease_information/ avian_influenza/affected_species_chart.jsp

van den Driessche P, Watmough J (2002) Reproduction numbers and sub-threshold endemic equilibria for compartmental models of disease transmission. Math Biosci 180:29-48

van der Goot JA, De Jong MCM, Koch G, van Boven M (2003) Comparison of the transmission characteristics of low and high pathogenicity avian influenza A virus (H5N2). Epidemiol Infect 131:1003-1013

van der Goot JA, De Jong MCM, Koch G, van Boven M (2005) Quantification of the effect of vaccination on transmission of avian influenza (H7N7) in chickens. Proc Natl Acad Sci USA 102:18141-18146

van der Goot JA, van Boven M, Stegeman A, van de Water SGPV, de Jong MCM, Koch G (2008) Transmission of highly pathogenic avian influenza $\mathrm{H} 5 \mathrm{~N} 1$ virus in Pekin ducks is significantly reduced by a genetically distant H5N2 vaccine. Virology 382:91-97

Verdugo C, Cardona CJ, Carpenter TE (2009) Simulation of an early warning system using sentinel birds to detect a change of a low pathogenic avian influenza virus (LPAIV) to high pathogenic avian influenza virus (HPAIV). Prev Vet Med 88:109-119

Ward MP, Maftei D, Apostu C, Suru A (2009) Estimation of the basic reproductive number $\left(\mathrm{R}_{0}\right)$ for epidemic, highly pathogenic avian influenza subtype H5N1 spread. Epidemiol Infect 137:219-226

Webster RG, Bean WJ, Gorman OT, Chambers TM, Kawaoka Y (1992) Evolution and ecology of influenza A viruses. Microbiol Rev 56:152-179

Webster RG, Peiris M, Chen H, Guan Y (2006) H5N1 outbreaks and enzootic influenza. Emerg Infect Dis 12:3-8

Webster RG, Krauss S, Hulse-Post D, Sturm-Ramirez K (2007) Evolution of influenza A viruses in wild birds. J Wildl Dis 43:S1-S6

White LJ, Cox MJ, Medley GF (1998) Cross immunity and vaccination against multiple microparasite strains. IMA J Math Appl Med 15:211-233 